\section{Estado nutricional de idosos no Brasil: uma abordagem multinível}

\author{
Nutritional status of elderly Brazilians: a multilevel \\ approach
}

\author{
Estado nutricional de ancianos en Brasil: un \\ enfoque multinivel
}

\section{Resumo}

O objetivo deste estudo foi diagnosticar o estado nutricional da população idosa brasileira, identificando fatores associados. Utilizou-se dados da Pesquisa de Orçamentos Familiares (POF 2008/2009) de 20.114 idosos, cujo perfil nutricional foi avaliado segundo o índice de massa corporal (IMC). As análises de fatores associados foram testadas com base no teste de qui-quadrado de Pearson e modelos lineares multiníveis. Na análise hierárquica constatou-se efeito significativo da Unidade da Federação na variância do IMC (valor de $p=0,001$ ). Em nível individual detectou-se associação negativa (valor de $p<0,001$ ) com: cor/raça amarela, sexo masculino, idosos que residiam sozinhos e idade; e positiva com a renda per capita. Observou-se ainda, maiores prevalências de baixo peso em idosos residentes no estrato rural (26,3\%) e nas regiões Nordeste (23,7\%) e Centro-oeste (20,9\%), e de obesidade em idosos que residem nas regiões Sul $(45,1 \%)$ e Sudeste (38,3\%) e no estrato urbano (39\%). Sugere-se aprofundar o estudo das características do estado nutricional de idosos utilizando-se variáveis contextuais.

Estado Nutricional; Índice de Massa Corporal;

Idoso; Inquéritos Demográficos
Ingrid Freitas da Silva Pereira 1 Maria Helena Constantino Spyrides 1 Lára de Melo Barbosa Andrade 1 


\section{Introdução}

Um dos maiores impactos que a transição demográfica produz diz respeito às mudanças na conformação da estrutura etária populacional e às implicações destas mudanças nas políticas sociais e econômicas. No Brasil, as quedas nos níveis de mortalidade em 1940 e, posteriormente, de natalidade por volta de 1960, resultaram em mudanças na configuração da pirâmide etária populacional, que deixou de ser predominantemente jovem, iniciando um processo progressivo de envelhecimento ${ }^{1}$.

Com o envelhecimento populacional e o aumento da expectativa de vida, verificaram-se mudanças no perfil epidemiológico da população, com o predomínio das doenças crônicas não transmissíveis (DCNT), específicas das faixas etárias mais avançadas, aumentando, cada vez mais, a necessidade do conhecimento dos fatores de risco que incidem sobre a prevalência das DCNT associadas à idade 2 .

Nesse contexto, o estado nutricional assume uma importante função na qualidade de vida e de saúde da população. Por um lado, a obesidade consolidou-se como agravo nutricional associado à alta incidência de DCNT, tais como doenças cardiovasculares, câncer e diabetes, influenciando, desta maneira, no perfil de morbimortalidade das populações 3 . Por outro, especificamente no grupo etário de idosos, a desnutrição apresenta-se fortemente associada ao aumento da incapacidade funcional, aumento no número de internações, redução da qualidade de vida, maior susceptibilidade às infecções e, consequentemente, aumento da mortalidade 4,5,6.

Dentre as várias ferramentas disponíveis para a avaliação do estado nutricional de idosos, sejam em estudos clínicos e principalmente em estudos populacionais, as medidas antropométricas apresentam-se como as mais utilizadas, tendo como destaque o emprego do índice de massa corporal (IMC). Apesar das limitações dos métodos de avaliação antropométrica e, especificamente do IMC, no sentido de não predizer a distribuição da gordura corporal e de não diferenciar massa magra de massa gorda, este é bastante utilizado pelo fato de produzir informações básicas das variações físicas dos indivíduos, possibilitando a classificação em graus de nutrição, além de ser um método não invasivo, de baixo custo, fácil e de rápida execução, e permite boa correlação com indicadores de morbimortalidade 7,8 .

A determinação do diagnóstico nutricional e a identificação dos fatores que contribuem para tal diagnóstico no indivíduo idoso são processos complexos, porém fundamentais para que políticas de intervenção específicas possam ser desenvolvidas. A complexidade se deve à ocorrência de diversas alterações, tanto fisiológicas quanto patológicas, peculiares do próprio envelhecimento, além de outros condicionantes sociais, econômicos e de estilo de vida, que podem interferir diretamente no estado nutricional de idosos 9 .

Entende-se que a investigação da situação nutricional de idosos, sob o olhar das diferenças demográficas e sociais das regiões do país, possa subsidiar ajustes nas políticas públicas de prevenção e promoção de saúde, a partir da compreensão destes fatores intervenientes nos agravos nutricionais. Nessa perspectiva, o presente trabalho tem por objetivo diagnosticar o estado nutricional e identificar fatores associados ao perfil nutricional na população idosa brasileira.

\section{Métodos}

Trata-se de um estudo transversal de base populacional, cuja população avaliada foi constituída por todos os indivíduos com idade igual ou superior a 60 anos, participantes da Pesquisa de Orçamentos Familiares (POF), 2008/2009, do Instituto Brasileiro de Geografia e Estatística (IBGE), totalizando 20.114 idosos 10 .

Com relação ao planejamento da amostra da POF, foram empregados procedimentos complexos de amostragem envolvendo estratificação geográfica e estatística do conjunto de setores censitários do país, sorteio de conglomerados de setores dentro de estratos e sorteio de domicílios dentro dos setores. Em seguida ao processo de seleção de setores e domicílios, os setores foram distribuídos ao longo dos quatro trimestres da pesquisa, garantindo que em todos eles os estratos geográfico e socioeconômico estivessem representados por meio dos domicílios selecionados.

De acordo com o planejamento amostral da POF, cada domicílio da amostra representa um determinado número de domicílios da população (universo) de onde esta amostra foi selecionada. Com isso, a cada domicílio da amostra está associado um peso amostral ou fator de expansão que, atribuído às características investigadas pela pesquisa, permite generalizar a informação da amostra para a população da qual foi extraída.

Para a avaliação do estado nutricional utilizou-se o IMC, calculado com base na divisão do peso em quilogramas $(\mathrm{kg})$ pela altura em metro (m) elevada ao quadrado $\left(\mathrm{kg} / \mathrm{m}^{2}\right)$.

Os dados de peso e altura foram submetidos, pelo próprio IBGE, a um tratamento de crítica e imputação, no qual foi utilizado o procedimento 
de imputação para tratar a não resposta e também os erros de resposta associados a valores rejeitados na etapa de crítica. No presente estudo, utilizaram-se os dados das variáveis de peso e altura já imputados.

As medidas antropométricas foram aferidas de todos os moradores encontrados nos domicílios durante o período da entrevista. Para a mensuração do peso foi utilizada uma balança eletrônica portátil com capacidade de $150 \mathrm{~kg}$ e graduação de $100 \mathrm{~g}$. A altura foi medida usandose estadiômetros portáteis, com trena retrátil, de extensão até $200 \mathrm{~cm}$ com precisão de $0,1 \mathrm{~cm}$.

A classificação do estado nutricional com base no IMC foi realizada segundo o critério proposto pela Nutrition Screening Initiative 11, que considera as modificações na composição corporal próprias do envelhecimento, sendo estes os pontos de corte adotados para idosos no Brasil segundo recomendações do Sistema de Vigilância Alimentar e Nutricional (SISVAN) 12. Nesse sentido, o estado nutricional foi classificado em baixo peso IMC $<22 \mathrm{~kg} / \mathrm{m}^{2}$, eutrofia IMC entre 22 e $27 \mathrm{~kg} / \mathrm{m}^{2}$ e sobrepeso IMC $>27 \mathrm{~kg} / \mathrm{m}^{2}$.

A variável dependente analisada no estudo foi o IMC e as variáveis independentes foram aquelas representadas pelas características sociodemográficas: sexo (masculino, feminino), faixa etária (60 a 69 anos: mais jovens, 70 a 79 anos: idosos-idosos, 80 anos e mais: longevos), cor/raça (branca, preta, amarela, parda, indígena), renda per capita em salários mínimos (até $1 / 4$, de $1 / 4$ a $1 / 2$, de $1 / 2$ a 1 , de 1 a 5,5 ou mais), situação do domicílio (urbano, rural), macrorregião (Norte, Nordeste, Sudeste, Sul, Centro-oeste), Unidades da Federação (Rondônia, Acre, Amazonas, Roraima, Amapá, Tocantins, Maranhão, Piauí, Ceará, Rio Grande do Norte, Paraíba, Pernambuco, Alagoas, Sergipe, Bahia, Minas Gerais, Espírito Santo, Rio de Janeiro, São Paulo, Paraná, Santa Catarina, Rio Grande do Sul, Mato Grosso do Sul, Mato Grosso, Distrito Federal), mora sozinho (sim, não), escolaridade (sem instrução, Ensino Fundamental e Médio, Ensino Superior e pós-graduação), pessoa de referência no domicílio (sim, não).

Para a classificação da renda familiar mensal per capita, dividiu-se o valor expresso na variável renda per capita da unidade de consumo pelo valor referente a um salário mínimo na data de referência da pesquisa (15 de janeiro de 2009), que era de $\mathrm{R} \$ 415,00$.

Para a análise dos dados, utilizou-se o software IBM SPSS versão 20 (IBM Corp., Armonk, Estados Unidos). Após a análise descritiva das variáveis, avaliou-se a relação das variáveis explicativas com a variável resposta (classificação do IMC) por meio do teste de associação qui-qua- drado de Pearson. O nível de significância estatística considerado foi de $5 \%$ ( $\mathrm{p}<0,05)$.

Considerando-se o desenho amostral da POF, que trata de uma amostra probabilística em dois estágios, com seleção primária de setores censitários e posterior seleção de domicílios, e supondo que indivíduos selecionados de uma mesma comunidade estão propensos a apresentar atitudes e comportamentos mais semelhantes do que aqueles selecionados em comunidades diferentes, tendo em vista que a forma como os indivíduos estão relacionados uns aos outros dentro dos grupos pode ser relevante para a compreensão da distribuição das condições de saúde 13, no presente trabalho foram aplicadas análises utilizando-se modelos de regressão hierárquicos ou multiníveis.

Diante de estruturas hierárquicas de dados, as observações tendem a ser correlacionadas, violando assim o pressuposto de independência assumido pelos modelos tradicionais. Ao ignorar a estrutura hierárquica, os modelos tradicionais produzem testes com erros-padrão dos parâmetros subestimados, aumentando a chance de seleção de variáveis explicativas, mesmo que estas não sejam estatisticamente significantes sobre o desfecho analisado 14,15 .

Nesse contexto, utilizou-se um modelo linear de efeitos mistos, buscando avaliar a consistência da influência de variáveis em nível individual (primeiro nível), assim como o diferencial entre as Unidades da Federação (segundo nível), perante as variações do IMC. As variáveis do primeiro nível, consideradas como efeitos fixos no estudo, foram: sexo (masculino, feminino); cor/ raça (branca, preta, amarela, parda, indígena); mora sozinho (sim, não); idade (anos); escolaridade (sem instrução, Ensino Fundamental e Médio, Ensino Superior e pós-graduação); renda mensal per capita (salário mínimo). Para efeito de modelagem, na análise multinível a variável a idade foi utilizada de forma contínua e as variáveis escolaridade e renda mensal per capita de forma ordinal.

Segundo Pinheiro \& Torres 16, indivíduos residentes na mesma Unidade da Federação tendem a apresentar comportamentos mais semelhantes no que se refere aos problemas de saúde, quando comparados àqueles residentes em outra Unidade da Federação, por serem submetidos ao mesmo contexto (condições socioeconômicas, fatores culturais, entre outros). Sendo assim, devido à heterogeneidade sociodemográfica, econômica e cultural existente entre essas Unidades da Federação, considerou-se a variável "Unidade da Federação” como efeito aleatório, buscando medir a influência que o estado onde o idoso vive pode exercer sobre o seu perfil nutricional. 
Visando a atender aos pressupostos de uma análise de variância, ou seja, normalidade e independência dos resíduos, com média zero e variância constante, na análise do modelo hierárquico trabalhou-se com a transformação logarítmica da variável IMC.

Com o intuito de avaliar a existência de diferencial de IMC médio entre as Unidades da Federação de cada macrorregião, aplicou-se a análise de variância (ANOVA), que testa a diferença de médias entre dois ou mais grupos com base na estatística F (F de Snedecor). Havendo diferença significante demonstrada pela ANOVA, aplicouse o teste de Tukey, que ao comparar simultaneamente todos os grupos dois a dois, identifica quais grupos assemelham ou diferem entre si. O nível de significância estatística considerado para todas as análises foi de $5 \%$.

\section{Resultados}

Do total de idosos entrevistados na pesquisa, a maioria era do sexo feminino $(55,6 \%)$, predominantemente da cor branca $(55,1 \%)$ ou parda (35,3\%). Com relação à faixa etária, como era de se esperar, com o avançar da idade, a representatividade porcentual tendeu a diminuir, registrando $56 \%$ de idosos mais jovens (60 a 69 anos), $30,8 \%$ de idosos-idosos (70 a 79 anos) e $13,2 \%$ de longevos (80 anos e mais) (Tabela 1).

Observou-se ainda o predomínio de idosos com renda mensal per capita entre 1 e 5 salários mínimos $(62,5 \%)$, residentes no estrato urbano do país $(82,7 \%)$ e com Ensino Fundamental e Médio (66,8\%). Quanto a ser pessoa de referência ou não na família, $63,7 \%$ declararam serem os responsáveis pela família, e $86,4 \%$ relataram não residir sozinhos (Tabela 1 ).

A prevalência de sobrepeso foi maior em idosos do sexo feminino $(41,9 \%)$ em comparação com o masculino (31,6\%), naqueles residentes no estrato urbano (39\%) contra $29,3 \%$ nos residentes na zona rural, bem como nos residentes nas regiões Sul $(45,1 \%)$ e Sudeste $(38,3 \%)$. Observou-se uma relação inversamente proporcional do sobrepeso com o aumento da faixa etária e diretamente proporcional com o aumento da renda per capita. O sobrepeso demonstrou ainda prevalência bastante expressiva em idosos de todas as cores/raça, com exceção para a cor amarela, que apresentou o menor porcentual (26\%) (Tabela 1).

Com relação ao baixo peso, este demonstrou relação diretamente proporcional com o avançar da idade, indicando que idosos mais longevos apresentaram maiores prevalências do déficit nutricional. Em contrapartida, encon- trou-se relação inversamente proporcional à escolaridade (com prevalência de $24 \%$ em idosos sem instrução contra $11 \%$ naqueles com Ensino Superior e pós-graduação) e à renda mensal per capita, na qual idosos com renda mensal de até 1/4 de salário mínimo registraram prevalência de 33,5\% e aqueles com 5 ou mais salários mínimos apresentaram prevalência de 11,3\% (Tabela 1).

O baixo peso ainda foi mais prevalente em idosos das cores amarela $(32,1 \%)$ e preta $(23,6 \%)$, residentes no estrato rural $(26,3 \%)$ e nas regiões Nordeste $(23,7 \%)$ e Centro-oeste $(20,9 \%)$. Os idosos que declararam morar sozinhos apresentaram maiores percentuais tanto de baixo peso (20,4\%) quanto de sobrepeso (38,5\%) (Tabela 1$)$.

A abordagem da análise multinível estimou a contribuição específica e simultânea de fatores individuais (primeiro nível), bem como a influência do diferencial por Unidades da Federação (efeito aleatório), na variância do IMC (Tabela 2). Verificou-se que, embora um porcentual pequeno da variação do IMC possa ser explicado mediante características e peculiaridades das distintas unidades federativas, foi constatada a significância estatística deste efeito aleatório. Fator que reforça a importância de se incorporar a estrutura hierárquica nos modelos de análises de estado nutricional.

Não foi constatado efeito significativo do nível de escolaridade. Para a variável cor/raça detectou-se associação negativa significativa para a amarela. Observa-se ainda que idosos do sexo masculino, bem como aqueles que relataram morar sozinhos, tenderam a apresentar menores valores de IMC em relação aos idosos de sexo feminino e daqueles que referiram não residir sozinhos (Tabela 2).

A variável idade esteve associada negativamente ao IMC, indicando que quanto maior a idade, menores são os valores de IMC. Já a variável renda mensal per capita apresentou consistente associação positiva com o IMC, cujo coeficiente positivo indica que quanto maior o nível de renda, maior o IMC do idoso (Tabela 2).

Os dados da análise das médias de IMC dos idosos por Unidades da Federação de cada macrorregião estão expostos na Tabela 3. Para a Região Nordeste, observou-se um diferencial quanto aos estados da Bahia, Maranhão e Piauí por apresentarem os menores valores de IMC, e nos estados de Pernambuco e Rio Grande do Norte pelas maiores médias de IMC da região.

Quanto à Região Centro-oeste, os destaques foram para Goiás e Mato Grosso do Sul que apresentaram menor e maior médias de IMC de idosos, respectivamente. A Região Norte demonstrou comportamento homogêneo entre todas as 
Tabela 1

Distribuição do estado nutricional de idosos segundo o índice de massa corporal (IMC). Brasil, 2009.

\begin{tabular}{|c|c|c|c|c|c|}
\hline \multirow[t]{2}{*}{ Variáveis } & \multirow{2}{*}{$\begin{array}{c}\text { Baixo peso } \\
(\%)\end{array}$} & \multirow{2}{*}{$\begin{array}{c}\text { Eutrofia } \\
(\%)\end{array}$} & \multirow{2}{*}{$\begin{array}{c}\text { obrepeso } \\
\text { (\%) }\end{array}$} & \multicolumn{2}{|c|}{ Total } \\
\hline & & & & $\mathrm{n}$ & $\%$ \\
\hline \multicolumn{6}{|l|}{ Sexo * } \\
\hline Masculino & 19,9 & 48,5 & 31,6 & 9.471 .374 & 44,4 \\
\hline Feminino & 18,2 & 39,9 & 41,9 & 11.847 .169 & 55,6 \\
\hline \multicolumn{6}{|l|}{ Cor/Raça * } \\
\hline Branca & 16,9 & 43,6 & 39,5 & 11.750 .726 & 55,1 \\
\hline Preta & 23,6 & 40,5 & 35,8 & 1.653 .415 & 7,8 \\
\hline Amarela & 32,1 & 41,9 & 26,0 & 214.920 & 1,0 \\
\hline Parda & 20,9 & 44,5 & 34,6 & 7.526 .130 & 35,3 \\
\hline Indígena & 9,2 & 51,1 & 39,7 & 106.137 & 0,5 \\
\hline Não sabe & 17,4 & 43,6 & 39,1 & 67.215 & 0,3 \\
\hline \multicolumn{6}{|l|}{ Faixa etária (anos) * } \\
\hline Mais jovens (60-69) & 16,6 & 43,7 & 39,7 & 11.947 .904 & 56,0 \\
\hline Idosos-idosos (70-79) & 20,1 & 43,6 & 36,2 & 6.565 .025 & 30,8 \\
\hline Longevos (80 e mais) & 26,3 & 43,8 & 29,9 & 2.805 .614 & 13,2 \\
\hline \multicolumn{6}{|c|}{ Renda mensal per capita (salários mínimos) * } \\
\hline até $1 / 4$ & 33,5 & 45,0 & 21,5 & 241.266 & 1,1 \\
\hline de $1 / 4$ a $1 / 2$ & 28,6 & 46,2 & 25,2 & 1.030 .412 & 4,8 \\
\hline de $1 / 2$ a 1 & 23,3 & 44,0 & 32,8 & 3.927 .981 & 18,4 \\
\hline de 1 a 5 & 18,3 & 42,2 & 39,5 & 13.324 .142 & 62,5 \\
\hline 5 ou mais & 11,3 & 49,3 & 39,0 & 2.794 .741 & 13,1 \\
\hline \multicolumn{6}{|l|}{ Situação de domicílio * } \\
\hline Urbano & 17,4 & 43,5 & 39,0 & 17.628 .300 & 82,7 \\
\hline Rural & 26,3 & 44,5 & 29,3 & 3.690 .243 & 17,3 \\
\hline \multicolumn{6}{|l|}{ Região * } \\
\hline Norte & 18,4 & 48,0 & 33,6 & 1.085 .685 & 5,1 \\
\hline Nordeste & 23,7 & 43,8 & 32,5 & 5.685 .106 & 26,7 \\
\hline Sudeste & 17,5 & 44,2 & 38,3 & 9.832 .219 & 46,1 \\
\hline Sul & 14,5 & 40,4 & 45,1 & 3.378 .798 & 15,8 \\
\hline Centro-oeste & 20,9 & 44,2 & 35,0 & 1.336 .736 & 6,3 \\
\hline \multicolumn{6}{|l|}{ Escolaridade * } \\
\hline Sem instrução & 24,0 & 44,3 & 31,7 & 5.444 .923 & 25,5 \\
\hline Ensino Fundamental e Médio & 18,0 & 43,6 & 38,5 & 14.275 .295 & 66,8 \\
\hline Ensino Superior e pós-graduação & 11,0 & 52,9 & 36,1 & 1.635 .743 & 7,7 \\
\hline \multicolumn{6}{|l|}{ Pessoa de referência * } \\
\hline Sim & 19,0 & 44,6 & 36,4 & 13.575 .359 & 63,7 \\
\hline Não & 18,9 & 42,1 & 39,0 & 7.743 .184 & 36,3 \\
\hline \multicolumn{6}{|l|}{ Mora sozinho * } \\
\hline Sim & 20,4 & 41,1 & 38,5 & 2.895 .188 & 13,6 \\
\hline Não & 18,7 & 44,1 & 37,2 & 18.423 .354 & 86,4 \\
\hline
\end{tabular}

Fonte: Pesquisa de Orçamentos Familiares (2008-2009) do Instituto Brasileiro de Geografia e Estatística (IBGE) 10.

* Valor de $p<0,001$ para teste de associação qui-quadrado de Pearson, com nível de significância menor que $5 \%(p<0,05)$. 
Modelo linear de efeitos mistos para o índice de massa corporal (IMC) de idosos, com transformação logarítmica. Brasil, 2009.

\begin{tabular}{|c|c|c|c|}
\hline Variáveis & Estimativa & Erro padrão & Valor de $\mathrm{p}$ \\
\hline Efeitos fixos & 3,3824 & 0,0135 & $<0,001$ \\
\hline \multicolumn{4}{|l|}{ Sexo } \\
\hline Masculino & $-0,0357$ & 0,0024 & $<0,001$ \\
\hline Feminino & - & - & - \\
\hline \multicolumn{4}{|l|}{ Cor/Raça } \\
\hline Branca & - & - & - \\
\hline Preta & 0,0007 & 0,0047 & 0,887 \\
\hline Amarela & $-0,0667$ & 0,0152 & $<0,001$ \\
\hline Parda & 0,0002 & 0,0028 & 0,934 \\
\hline Indígena & $-0,0052$ & 0,0149 & 0,728 \\
\hline \multicolumn{4}{|l|}{ Mora sozinho } \\
\hline Sim & $-0,0168$ & 0,0037 & $<0,001$ \\
\hline Não & - & - & - \\
\hline Idade (anos) & $-0,0030$ & 0,0002 & $<0,001$ \\
\hline Escolaridade * & 0,0038 & 0,0024 & 0,117 \\
\hline Renda mensal per capita (salário mínimo) & 0,0226 & 0,0017 & $<0,001$ \\
\hline \multicolumn{4}{|l|}{ Efeitos aleatórios } \\
\hline Residual & 0,0296 & 0,0003 & $<0,001$ \\
\hline Unidades da Federação & 0,0004 & 0,0001 & 0,001 \\
\hline
\end{tabular}

Fonte: Pesquisa de Orçamentos Familiares (2008-2009) do Instituto Brasileiro de Geografia e Estatística (IBGE) 10.

* Sem instrução, Ensino Fundamental e Médio, Ensino Superior e pós-graduação.

Unidades da Federação, podendo-se inferir que as médias de IMC dos idosos de todos os estados desta região são, praticamente, iguais.

Para a Região Sudeste, os estados de Minas Gerais e Espírito Santo não apresentaram diferença significativa em suas médias de IMC, o que também ocorreu nos estados do Rio de Janeiro e São Paulo. Já em relação à Região Sul, o Paraná registrou a menor média de IMC, diferentemente do Rio Grande do Sul, onde foram encontrados os maiores valores do índice.

Quando realizada a análise por sexo, observase que em todos os estados as médias entre as mulheres foram maiores do que as dos homens, destacando-se as médias dos três estados da Região Sul e a de Rondônia na Região Norte.

No geral, na análise por sexo, o comportamento nas Unidades da Federação dentro de cada região não variou muito em relação à análise do total de idosos. As principais variações foram: das mulheres do Rio Grande do Norte, que deixaram de estar no grupo das maiores médias da Região Nordeste; a diferenciação entre as Unidades da Federação de médias de IMC dos homens na Região Norte, com o Acre apresentando os menores valores e o Amapá os mais elevados índices, fato que não ocorreu entre as médias de
IMC do total de idosos e nem do sexo feminino; na Região Sul a análise diferenciada por sexo não demonstrou diferença significativa entre as médias de IMC dos três estados para ambos os sexos.

\section{Discussão}

Os resultados do presente estudo evidenciaram que o sobrepeso é mais prevalente entre as idosas. Em contrapartida, o baixo peso esteve mais prevalente entre os idosos do sexo masculino. Esses resultados estão em consonância com os achados de estudos desenvolvidos com grupos específicos de idosos em algumas cidades do país, como: São Paulo 7, Pinhais (Paraná) 17, Londrina (Paraná) 18, Bambuí (Minas Gerais) 19, Fortaleza (Ceará) 20 e Campina Grande (Paraíba) 21 , bem como em trabalhos desenvolvidos na Itália 22, México 23 e Chile 24.

O diferencial por sexo do perfil de estado nutricional demonstrado neste trabalho pode advir de uma explicação de ordem fisiológica. O processo de envelhecimento é acompanhado por diversas alterações, dentre as quais ocorrem mudanças na composição corporal 
Média de índice de massa corporal (IMC) de idosos por Unidades da Federação, segundo o sexo. Brasil, 2009.

\begin{tabular}{|c|c|c|c|}
\hline \multirow[t]{2}{*}{ Regiões/Unidades da Federação } & \multicolumn{3}{|c|}{ Média de IMC } \\
\hline & Total & Masculina & Feminina \\
\hline \multicolumn{4}{|l|}{ Nordeste } \\
\hline Bahia & 24,78 a & 23,98 a & 25,43 a \\
\hline Piauí & 25,13 a & 24,36 ab & $25,80 \mathrm{ab}$ \\
\hline Maranhão & 25,10 a & $24,86 a b c$ & 25,30 a \\
\hline Ceará & $25,33 \mathrm{ab}$ & $24,94 a b c$ & $25,64 a b c$ \\
\hline Alagoas & $25,42 a b c$ & $24,98 \mathrm{bc}$ & $25,79 a b c$ \\
\hline Sergipe & $26,02 \mathrm{bcd}$ & $25,28 \mathrm{bc}$ & $26,60 \mathrm{bcd}$ \\
\hline Paraíba & $26,15 \mathrm{~cd}$ & 25,25 bc & $26,88 d$ \\
\hline Pernambuco & $26,20 d$ & $25,20 \mathrm{bc}$ & $26,87 d$ \\
\hline Rio Grande do Norte & $26,31 d$ & $25,63 c$ & $26,73 \mathrm{~cd}$ \\
\hline \multicolumn{4}{|l|}{ Centro-oeste } \\
\hline Goiás & 25,15 a & 24,78 a & 25,49 a \\
\hline Mato Grosso & $25,45 \mathrm{ab}$ & 25,25 a & 25,67 a \\
\hline Distrito Federal & $25,92 \mathrm{bc}$ & $25,61 \mathrm{ab}$ & 26,16 ab \\
\hline Mato Grosso do Sul & $26,56 c$ & $26,25 b$ & $26,89 b$ \\
\hline \multicolumn{4}{|l|}{ Norte } \\
\hline Acre & 25,42 a & 24,61 a & 26,24 a \\
\hline Amazonas & 25,58 a & $25,52 \mathrm{ab}$ & 25,65 a \\
\hline Pará & 25,59 a & $24,88 \mathrm{ab}$ & 26,28 a \\
\hline Tocantins & 25,64 a & $25,02 \mathrm{ab}$ & 26,35 a \\
\hline Roraima & 26,16 a & $26,20 \mathrm{~b}$ & 26,13 a \\
\hline Rondônia & 26,27 a & $25,22 \mathrm{ab}$ & 27,33 a \\
\hline Amapá & 26,52 a & $26,18 b$ & 26,88 a \\
\hline \multicolumn{4}{|l|}{ Sudeste } \\
\hline Minas Gerais & 25,46 a & $24,90 a$ & 25,46 a \\
\hline Espírito Santo & 25,66 a & $25,36 \mathrm{ab}$ & 25,66 a \\
\hline Rio de Janeiro & $26,36 b$ & $25,46 \mathrm{ab}$ & $26,36 b$ \\
\hline São Paulo & $26,49 \mathrm{~b}$ & $25,79 \mathrm{~b}$ & $26,49 \mathrm{~b}$ \\
\hline \multicolumn{4}{|l|}{ Sul } \\
\hline Paraná & 26,44 a & 25,78 a & 27,00 a \\
\hline Santa Catarina & $26,84 a b$ & 26,38 a & 27,23 a \\
\hline Rio Grande do Sul & $27,08 \mathrm{~b}$ & 26,40 a & 27,63 a \\
\hline
\end{tabular}

Fonte: Pesquisa de Orçamentos Familiares (2008-2009) do Instituto Brasileiro de Geografia e Estatística (IBGE) 10.

* Letras iguais indicam que não há diferença significante no nível de 5\% para o teste de Tukey entre as médias de IMC de cada macrorregião, para o total de indivíduos ou segundo o sexo.

dos indivíduos. Nesse contexto, há não só um aumento progressivo na deposição da gordura corporal, como também a sua redistribuição, sendo observada uma diminuição na região dos membros e um maior acúmulo na região abdominal. Geralmente, essa deposição ocorre mais precocemente nos homens, provavelmente por volta da meia-idade, e mais tardiamente nas mulheres, após o período da menopausa 25. Na menopausa, o declínio da função ovariana induzido pela depleção estrogênica provo- caria aumento nos níveis de adiposidade total e conformação de um padrão centralizado de massa gorda 26 .

Com relação à faixa etária, esta esteve associada positiva e diretamente com o baixo peso e inversamente com o sobrepeso. Nascimento et al. 27, ao avaliarem o estado nutricional de idosos residentes no Município de Viçosa (Minas Gerais), também identificaram redução significativa do IMC com o aumento da faixa etária. Corroborando ainda com esses resultados, 
Santos \& Sichieri 28 também relataram menores prevalências de sobrepeso quanto maior a faixa etária de idosos participantes da Pesquisa de Saúde e Nutrição no Município do Rio de Janeiro. Menezes et al. 21 também registraram a mesma relação observada no presente trabalho entre estado nutricional e idade, em estudo de base populacional realizado em Campina Grande.

A redução do percentual de sobrepeso com o avanço do envelhecimento pode ser explicada, em parte, pelo efeito do viés de sobrevivência, devido à interferência do excesso de peso e das morbidades a este associadas, no aumento da mortalidade em idosos obesos antes de atingirem 80 anos 18 .

Já a relação entre ser mais velho e apresentar baixo peso advém, possivelmente, das próprias transformações peculiares à progressão do processo de envelhecimento, que desencadeia diversas alterações de ordem fisiológica, patológica e psicológica que afetam diretamente o estado nutricional do indivíduo, tais como redução do olfato e da visão, diminuição dos botões gustativos, dificuldades de mastigação e constipação intestinal devido à redução da motilidade, bem como diminuição da capacidade cognitiva e funcional 29,30 .

Além das alterações que dificultam a ingestão alimentar adequada durante o envelhecimento, em parte devido ao sedentarismo muito comum em idosos, o processo de regeneração muscular se torna mais difícil, ocasionando a perda progressiva da massa muscular esquelética. Essa condição tem importantes implicações no estado nutricional e na saúde dos idosos, contribuindo para a fragilidade, perda funcional, dependência, deficiência, aumento de morbidades e morte prematura 31 .

É importante considerar a magnitude do baixo peso na população idosa no Brasil, ainda que, diferentemente da obesidade, sua prevalência seja menor e venha apresentando tendência de declínio; o baixo peso ainda é bastante incidente neste segmento populacional, contribuindo para o aumento da mortalidade, principalmente em grupos de maior limitação econômica 32. Independentemente da fisiopatologia, o problema dos óbitos por desnutrição em idosos traz à tona problemas sociais relacionados à desigualdade de acesso à alimentação em quantidade e qualidade satisfatórias e ao papel das políticas públicas 33 .

O contexto socioeconômico revela o Brasil como um país extremamente desigual. Essa iniquidade pode ser demonstrada com base em comparações entre as grandes regiões do país. No Nordeste, $68 \%$ dos idosos residem em domicílios com renda familiar per capita menor que 1 salário mínimo, no Sul e no Sudeste este percentual está abaixo de $35 \% 34$.

As condições socioeconômicas desempenham um papel fundamental em relação à qualidade de vida na velhice. A elevação da renda, da escolaridade, das condições de moradia e maior acesso aos bens e serviços interferem na disponibilidade e/ou acesso aos alimentos, afetando as escolhas dietéticas e o padrão alimentar ao longo da vida 35 .

Os achados deste estudo indicaram associação positiva do IMC com a renda mensal per capita na análise dos modelos hierárquicos. Campos et al. 36 encontraram resultados diferentes dos expostos neste trabalho, nos quais ao avaliarem o estado nutricional de idosos baseandose em dados da Pesquisa sobre Padrões de Vida (PPV) segundo a classificação da Organização Mundial da Saúde (OMS) 37, identificaram que os idosos com renda domiciliar entre 2 e 5 salá rios mínimos apresentaram maiores chances de desenvolver o baixo peso, quando comparados àqueles com renda domiciliar menor que 2 salários mínimos. Já para o sobrepeso e a obesidade, o aumento da chance em apresentá-los foi proporcional ao aumento da renda.

Similarmente ao ocorrido com a renda, e possivelmente devido à íntima relação entre renda e escolaridade, neste estudo foram encontradas maiores prevalências de baixo peso quanto menor o nível educacional dos idosos. Contudo, as prevalências de sobrepeso mostraram-se semelhantes entre os diferentes níveis de escolaridade, o que pode ter influenciado a não associação da escolaridade com a variabilidade do IMC na aplicação do modelo hierárquico. Barreto et al. 19 ao investigarem o IMC e fatores relacionados em 1.451 idosos de Bambuí, identificaram associação inversa entre o IMC e nível educacional, demonstrada pelas maiores razões de chance de apresentar baixo peso e menores razões de chance de apresentar obesidade em idosos com mais anos de estudos.

Ainda sobre a relação entre obesidade e nível de renda e escolaridade, Silveira et al. 38, ao avaliarem a prevalência de obesidade em idosos residentes em Pelotas (Rio Grande do Sul), segundo dois diferentes pontos de corte do IMC, constataram, independentemente do desfecho analisado, maiores prevalências de obesidade nas categorias de mais baixo quartil de renda per capita, bem como naqueles com menos de um ano de escolaridade.

Em um estudo similar a este, porém usando como ponto de corte para o sobrepeso o IMC $\geq$ $25,0 \mathrm{~kg} / \mathrm{m}^{2}$, Silva et al. ${ }^{39}$, utilizando dados da POF 2002/2003, encontraram as mesmas associações da variável IMC quanto à renda e escolaridade. 
Os autores destacam a violência urbana como um possível fator de inibição da prática de atividade física no lazer em idosos de renda elevada, levando-os a optar por atividades em que não seja necessário sair de casa, atitudes que contribuem com valores aumentados de IMC.

Outro aspecto relevante a ser destacado diz respeito aos maiores porcentuais tanto de baixo peso quanto de sobrepeso entre os idosos que relataram morar sozinhos. Mesmo não representando a maioria entre os arranjos familiares de idosos no Brasil, tem crescido consideravelmente o percentual daqueles que vivem sozinhos. A necessidade de auxílio, seja este físico, financeiro ou afetivo, faz com que muitos idosos ainda deixem de optar por viver de forma independente para morar com suas famílias. Seguindo esse pensamento, esperava-se que idosos que moram sozinhos apresentassem melhores condições de saúde 40 .

Contudo, por não terem alguém que os ajudem em suas atividades rotineiras, sirva de companhia e lhes dediquem cuidados, o fato de morar só vem sendo associado a um decréscimo na qualidade de vida e a um agravamento da morbidade, constituindo, até mesmo, um indicador de risco de mortalidade 41 . Sendo assim, idosos que residem sozinhos estariam mais vulneráveis a apresentar distúrbios nutricionais, sejam estes de déficit ou de excesso.

A relação entre o baixo peso do idoso e a condição de viver só já foi evidenciada em alguns estudos nacionais e internacionais, pautandose na possível justificativa de que idosos que vivem só tendem a apresentar mais problemas de saúde que podem repercutir em alterações no apetite e na aquisição de alimentos, predispondo o indivíduo à desnutrição 30,35,42. Entretanto, o fato de morar sozinho também pode influenciar nos valores elevados de IMC, no sentido de que estes idosos tenderiam a consumir alimentos de baixa qualidade nutricional, induzindo ao sobrepeso 43 .

A distribuição geográfica dos distúrbios nutricionais de idosos no Brasil revelou predominância de baixo peso nas regiões Nordeste e Centro-oeste e no estrato rural. Em contrapartida, o sobrepeso esteve mais presente no estrato urbano e nas regiões Sul e Sudeste.

Considerando a magreza como IMC $<18,5 \mathrm{~kg} /$ $\mathrm{m}^{2}$ e o sobrepeso por IMC $\geq 25 \mathrm{~kg} / \mathrm{m}^{2}$, Tavares \& Anjos 44, utilizando dados da Pesquisa Nacional de Saúde e Nutrição (PNSN) de 1989, encontraram a mesma tendência de distribuição espacial do estado nutricional de idosos no Brasil, há 20 anos, em relação aos dados utilizados neste estudo. Foram observadas maiores prevalências de magreza nas regiões Nordeste e Centro-oeste, para ambos os sexos nas respectivas regiões, sendo $8,8 \%$ e $10,7 \%$ para o sexo masculino e $11,9 \%$ e $11,6 \%$ para o sexo feminino. Maiores percentuais de magreza nas áreas rurais também foram observadas para ambos os sexos, com exceção do grupo de mulheres do estrato rural da Região Sul. Quanto ao sobrepeso, Tavares \& Anjos 44 também constataram maiores prevalências no estrato urbano e nas regiões Sul e Sudeste. Mais recentemente, utilizando dados da POF 200/-2003 e os mesmos critérios de classificação usados por Tavares \& Anjos 44, Silva et al. 39 encontraram a mesma distribuição regional do baixo peso em idosos, porém, para o sobrepeso, as regiões que apresentaram maiores porcentuais foram Sul e Centro-oeste.

A espacialização regional e por Unidades da Federação da situação nutricional no Brasil acompanha a heterogeneidade socioeconômica do país. Um aspecto importante na compreensão do cenário epidemiológico dos problemas alimentares/nutricionais se configura nas disparidades de distribuição de renda, tanto entre regiões quanto entre o meio rural (bem mais pobre) e o urbano 45 . Desse modo, as características ambientais, culturais e socioeconômicas podem repercutir no estilo de vida, assim como na disponibilidade e/ou acesso aos alimentos, o que parece explicar a divergência quanto aos fatores associados ao estado nutricional em distintos municípios, estados ou regiões 35 .

Uma possível limitação do presente trabalho refere-se ao próprio delineamento de pesquisa da POF, que ao investigar apenas domicílios particulares permanentes, deixa de considerar a situação de vida de uma parcela importante do segmento populacional de idosos, referente àqueles que estão institucionalizados. Mesmo assim, a relevância desses achados é reforçada pela representatividade da amostra utilizada na pesquisa, que permite que os resultados possam ser extrapolados para toda a população de idosos do Brasil, contribuindo com o conhecimento ampliado da situação nutricional dos idosos em todo o país e segundo características regionais e sociais, abrangência normalmente escassa nas pesquisas da área.

Reitera-se a necessidade de se estudar a situação nutricional da população idosa mediante as condições contextuais que a rodeiam e não somente tomando características individuais. Somente a partir dessa compreensão é que se poderá ter um conhecimento aprofundado dos fatores determinantes do estado nutricional de idosos, que está diretamente relacionado ao perfil epidemiológico e de mortalidade deste segmento populacional. E, mediante essas informações, práticas de monitoramento e de 
promoção da saúde e qualidade de vida poderão ser implementadas de maneira específica e eficaz, impulsionando o crescimento em diver- sos setores da sociedade, como o econômico, de saúde e da previdência social.

\section{Colaboradores}

I. F. S. Pereira e M. H. C. Spyrides participaram de todas as etapas da pesquisa, desde o planejamento, interpretação dos dados, elaboração e revisão final do artigo. L. M. B. Andrade colaborou na interpretação dos dados e revisão da versão final do artigo.

\section{Referências}

1. Vasconcelos AMN, Gomes MMF. Transição demográfica: a experiência brasileira. Epidemiol Serv Saúde 2012; 21:539-48.

2. Campolina AG, Adami F, Santos JLF, Lebrão ML. A transição de saúde e as mudanças na expectativa de vida saudável da população idosa: possíveis impactos da prevenção de doenças crônicas. Cad Saúde Pública 2013; 29:1217-29.

3. Tomasi E, Nunes BP, Thumé E, Silveira DS, Siqueira FV, Piccini RX, et al. Utilização de serviços de saúde no Brasil: associação com indicadores de excesso de peso e gordura abdominal. Cad Saúde Pública 2014; 30:1515-24.

4. Almeida MF, Marucci MFN, Gobbo LA, Ferreira LS, Dourado DAQS, Duarte YAO, et al. Anthropometric changes in the Brazilian cohort of older adults: SABE Survey (Health, Well-Being, and Aging). J Obes 2013; 2013:695496.

5. Strobl R, Müller M, Emeny R, Peters A, Grill E. Distribution and determinants of functioning and disability in aged adults: results from the German KORA-Age study. BMC Public Health 2013, 13: 137-47.
6. Souza KT, Mesquita LAS, Pereira LA, Azeredo CM Baixo peso e dependência funcional em idosos institucionalizados de Uberlândia - MG, Brasil. Ciênc Saúde Coletiva 2014; 19:3513-20.

7. Barbosa AR, Souza JMP, Lebrão ML, Laurenti R, Marucci MFN. Anthropometry of elderly residents in the city of São Paulo, Brazil. Cad Saúde Pública 2005; 21:1929-38.

8. Cortez ACL, Martins MCC. Indicadores antropométricos do estado nutricional em idosos: uma revisão sistemática. UNOPAR Cient Ciênc Biol Saúde 2012; 14:271-7.

9. Ferreira AA, Menezes MFG, Tavares EL, Nunes NC, Souza FP, Albuquerque NAF, et al. Estado nutricional e autopercepção da imagem corporal de idosas de uma Universidade Aberta da Terceira Idade. Rev Bras Geriatr Gerontol 2014; 17:289-301.

10. Instituto Brasileiro de Geografia e Estatística. Pesquisa de Orçamentos Familiares 2008-2009: antropometria e estado nutricional de crianças, adolescentes e adultos no Brasil. Rio de Janeiro: Instituto Brasileiro de Geografia e Estatística; 2010. 
11. Nutrition Screening Initiative. Incorporating nutrition screening and interventions into medical practice: a monograph for physicians. Washington DC: American Academy of Family Physicians/ American Dietetic Association/National Council on Aging Inc.; 1994.

12. Coordenação-Geral de Alimentação e Nutrição, Departamento de Atenção Básica, Secretaria de Atenção à Saúde, Ministério da Saúde. Orientações para a coleta e análise de dados antropométricos em serviços de saúde: norma técnica do sistema de vigilância alimentar e nutricional - SISVAN Brasília: Ministério da Saúde, 2011. (Série G. Estatística e Informação em Saúde).

13. Diez-Roux AV. Multilevel analysis in public health research. Annu Rev Public Health 2000; 21:171-92.

14. Goldstein H. Multilevel statistical models. 2nd Ed. London: Arnold; 1995.

15. Pinheiro JC, Bates DM. Mixed-effects models in Sand S-PLUS. New York: Springer-Verlag; 2000.

16. Pinheiro RS, Torres TZG. Uso de serviços odontológicos entre os estados do Brasil. Ciênc Saúde Coletiva 2006; 11:999-1010.

17. Bassler TC, Lei DLM. Diagnóstico e monitoramento da situação nutricional da população idosa em município da região metropolitana de Curitiba (PR). Rev Nutr 2008; 21:311-21.

18. Cabrera MAS, Jacob Filho W. Obesidade em idosos: prevalência, distribuição e associação com hábitos e co-morbidades. Arq Bras Endocrinol Metab 2001; 45:494-501.

19. Barreto SM, Passos VMA, Lima-Costa MFF. Obesity and underweight among Brazilian elderly. The Bambuí Health and Aging Study. Cad Saúde Pública 2003; 19:605-12.

20. Menezes TN, Souza JMP, Marucci MFN. Avaliação do estado nutricional dos idosos residentes em Fortaleza-CE: o uso de diferentes indicadores antropométricos. Rev Bras Cineantropom Desempenho Hum 2008; 10:315-22.

21. Menezes TN, Brito MT, Araújo TBP, Silva CCM, Nolasco RRN, Fischer MATS. Perfil antropométrico dos idosos residentes em Campina Grande - PB. Rev Bras Geriatr Gerontol 2013; 16:19-27.

22. Perissinotto E, Pisent C, Sergi G, Grigoletto F, Enzi G. Anthropometric measurements in the elderly: age and gender differences. Br J Nutr 2002; 87: 177-86.

23. Velásquez-Alva MC, Irigoven ME, Zepeda M, Sánchez VM, Garcia CMP, Castillo LM. Anthropometric measurements of a sixty-year and older Mexican urban group. J. Nutr Health Aging 2004; 8: 350-4.

24. Santos JL, Albala C, Lera L, García C, Arroyo P, Pérez-Bravo F, et al. Anthropometric measurements in the elderly population of Santiago, Chile. Nutrition 2004; 20:452-7.

25. Marucci MFN, Barbosa AR. Estado nutricional e capacidade física. In: Lebrão ML, Duarte YAO, organizadores. SABE - Saúde, Bem-estar e Envelhecimento. O projeto SABE no município de São Paulo: uma abordagem inicial. Brasília: Organização Pan-Americana da Saúde; 2003. p. 95-117.
26. Rocha JSB, Ogando BMA, Reis VMCP, Ávila WRM, Carneiro AG, Gabriel RECD, et al. Impacto de um programa de exercício físico na adiposidade e na condição muscular de mulheres pós-menopáusicas. Rev Bras Ginecol Obstet 2012; 34:414-9.

27. Nascimento CM, Ribeiro AQ, Cotta RMM, Acurcio FA, Peixoto SV, Priore SE, et al. Estado nutricional e fatores associados em idosos do Município de Viçosa, Minas Gerais, Brasil. Cad Saúde Pública 2011; 27:2409-18.

28. Santos DM, Sichieri R. Índice de massa corporal e indicadores antropométricos de adiposidade em idosos. Rev Saúde Pública 2005; 39:163-8.

29. Garcia ANM, Romani SAM, Lira PIC. Indicadores antropométricos na avaliação nutricional de idosos: um estudo comparativo. Rev Nutr 2007; 20:371-8.

30. Schilp J, Wijnhoven AH, Deeg DJH, Vissert M. Early determinants for the development of undernutrition in an older general population: Longitudinal Aging Study Amsterdam. Br J Nutr 2011; 106: 708-17.

31. Leite LEA, Resende TL, Nogueira GM, Cruz IBM, Schneider RH, Gottlieb MGV. Envelhecimento, estresse oxidativo e sarcopenia: uma abordagem sistêmica. Rev Bras Geriatr Gerontol 2012; 15:365-80.

32. Monteiro CA, Mondine L, Souza ALM, Popkin BM. Da desnutrição para a obesidade: a transição nutricional no Brasil. In: Monteiro CA, organizador. Velhos e novos males da saúde no Brasil: a evolução do país e suas doenças. 2a Ed. São Paulo: Editora Hucitec; 2000. p. 247-55.

33. Otero UB, Rozenfeld S, Gadelha AMJ, Carvalho MS. Mortalidade por desnutrição em idosos, Região Sudeste do Brasil, 1980-1997. Rev Saúde Pública 2002; 36:141-8.

34. Geib LTC. Determinantes sociais da saúde do idoso. Ciênc Saúde Coletiva 2012; 17:123-33.

35. Fares D, Barbosa AR, Borgatto AF, Coqueiro RS, Fernandes MH. Fatores associados ao estado nutricional de idosos de duas regiões do Brasil. Rev Assoc Méd Bras 2012; 58:434-41.

36. Campos MAG, Pedroso ERP, Lamounier JA, Colosimo EA, Abrantes MM. Estado nutricional e fatores associados em idosos. Rev Assoc Méd Bras 2006; 52:214-21.

37. World Health Organization. Obesity: preventing and managing the global epidemic. Geneva: World Health Organization; 1998. (Technical Report Series, 894).

38. Silveira EA, Kac G, Barbosa LS. Prevalência e fatores associados à obesidade em idosos residentes em Pelotas, Rio Grande do Sul, Brasil: classificação da obesidade segundo dois pontos de corte do índice de massa corporal. Cad Saúde Pública 2009; 25:1569-77.

39. Silva VS, Souza I, Petroski EL, Silva DAS. Prevalência e fatores associados ao excesso de peso em idosos brasileiros. Rev Bras Ativ Fís Saúde 2011; 16:289-94 
40. Camargos MCS, Rodrigues RN, Machado CJ. Idoso, família e domicílio: uma revisão narrativa sobre a decisão de morar sozinho. Rev Bras Estud Popul 2011; 28:217-30.

41. Dias DSG, Carvalho CS, Araújo CV. Comparação da percepção subjetiva de qualidade de vida e bemestar de idosos que vivem sozinhos, com a família e institucionalizados. Rev Bras Geriatr Gerontol 2013; 16:127-38.

42. Ferry M, Sidobre B, Lambertin A, Barberger-Gateau P. The solinut study: analysis of the interaction between nutrition and loneliness in persons aged over 70 years. J Nutr Health Aging 2005; 9:261-8.

\section{Abstract}

The objectives of this study were to diagnose the nutritional status of the elderly Brazilian population and to identify associated factors. The study used data from the Brazilian Household Budget Survey (2008/2009) for 20,114 elderly, whose nutritional status was assessed by body mass index (BMI). Associated factors were tested with the Pearson chi-square test and multilevel linear models. The hierarchical analysis showed a significant effect of state of Brazil on BMI variance ( $p$ value $=0.001)$. The individual level showed a negative association ( $p$-value $<0.001$ ) with Asian-descendant race, male gender, living alone, and older age and a positive association with per capita income. Underweight was more prevalent among elderly in rural areas (26.3\%) and in the Northeast (23.7\%) and Central regions $(20.9 \%)$, and obesity was more prevalent in the South (45.1\%) and Southeast (38.3\%) and in cities (39\%). The study suggests the importance of further indepth research on nutritional status of elderly based on contextual variables.

Nutritional Status; Body Mass Index; Aged; Population Surveys
43. Ledikwe JH, Smiciklas-Wright H, Jensen GL, Friedmann JM, Strill CD. Nutritional risk assessment and obesity in rural older adults: a sex difference. Am J Clin Nutr 2003; 77:551-8.

44. Tavares EL, Anjos LA. Perfil antropométrico da população idosa brasileira. Resultados da Pesquisa Nacional sobre Saúde e Nutrição. Cad Saúde Pública 1999; 15:759-68.

45. Batista Filho M, Rissin A. A transição nutricional no Brasil: tendências regionais e temporais. Cad Saúde Pública 2003; 19 Suppl 1:S181-91.

\section{Resumen}

El objetivo de este estudio fue diagnosticar el estado nutricional de la población anciana brasileña, identificando factores asociados. Se utilizaron los datos de 20.114 ancianos, procedentes de la Investigación sobre Presupuestos Familiares (2008/2009), cuyo perfil nutricional fue evaluado según el índice de masa corporal (IMC). Los análisis de factores asociados se probaron a partir del test chi-cuadrado de Pearson y modelos lineales multiniveles. En el análisis jerárquico se constató el efecto significativo de la Unidad de la Federación en la variancia del IMC (valor de $p=0,001$ ). En un nivel individual, se detectó una asociación negativa (valor de $p<0,001$ ) con: color/raza oriental, sexo masculino, ancianos que residian solos y con la edad; y positiva con la renta per cápita. Se observaron incluso mayores prevalencias de bajo peso en ancianos residentes en el estrato rural $(26,3 \%)$ y en la regiones Nordeste $(23,7 \%)$ y Centro-oeste (20,9\%), y de obesidad en ancianos residentes en las regiones Sur $(45,1 \%)$ y Sudeste $(38,3 \%)$ y en el estrato urbano (39\%). Se sugiere profundizar en el estudio de las características del estado nutricional de ancianos, utilizando variables contextuales.

Estado Nutricional; Índice de Masa Coporal; Anciano; Encuestas Demográficas
Recebido em 01/Dez/2014

Versão final reapresentada em 16/Ago/2015

Aprovado em 22/Fev/2016 